\title{
Conservation of structure and activity in Plasmodium purine nucleoside phosphorylases Apirat Chaikuad ${ }^{1,2}$ and R Leo Brady*1
}

Address: ${ }^{1}$ Department of Biochemistry, University of Bristol, Bristol, BS8 1TD, UK and ${ }^{2}$ Current address: Oxford Structural Genomics Consortium, Oxford, UK

Email: Apirat Chaikuad - Apirat.Chaikuad@sgc.ox.ac.uk; R Leo Brady* - L.Brady@bris.ac.uk

* Corresponding author

Published: 3 July 2009

BMC Structural Biology 2009, 9:42 doi:10.1 186/1472-6807-9-42

This article is available from: http://www.biomedcentral.com/I472-6807/9/42

(c) 2009 Chaikuad and Brady; licensee BioMed Central Ltd.

This is an Open Access article distributed under the terms of the Creative Commons Attribution License (http://creativecommons.org/licenses/by/2.0), which permits unrestricted use, distribution, and reproduction in any medium, provided the original work is properly cited.
Received: 26 February 2009

Accepted: 3 July 2009

\begin{abstract}
Background: Purine nucleoside phosphorylase (PNP) is central to purine salvage mechanisms in Plasmodium parasites, the causative agents of malaria. Most human malaria results from infection either by Plasmodium falciparum (Pf), the deadliest form of the parasite, or by the widespread Plasmodium vivax (Pv). Whereas the PNP enzyme from Pf has previously been studied in detail, despite the prevalence of $P v$ little is known about many of the key metabolic enzymes from this parasite, including PvPNP.
\end{abstract}

Results: The crystal structure of PvPNP is described and is seen to have many features in common with the previously reported structure of PfPNP. In particular, the composition and conformations of the active site regions are virtually identical. The crystal structure of a complex of PfPNP cocrystallised with inosine and arsenate is also described, and is found to contain a mixture of products and reactants - hypoxanthine, ribose and arsenate. The ribose $\mathrm{Cl}^{\prime}$ in this hybrid complex lies close to the expected point of symmetry along the PNP reaction coordinate, consistent with a conformation between the transition and product states. These two Plasmodium PNP structures confirm the similarity of structure and mechanism of these enzymes, which are also confirmed in enzyme kinetic assays using an array of substrates. These reveal an unusual form of substrate activation by 2'-deoxyinosine of PvPNP, but not PfPNP.

Conclusion: The close similarity of the Pf and Pv PNP structures allows characteristic features to be identified that differentiate the Apicomplexa PNPs from the human host enzyme. This similarity also suggests there should be a high level of cross-reactivity for compounds designed to inhibit either of these molecular targets. However, despite these similarities, there are also small differences in the activities of the two Plasmodium enzymes.

\section{Background}

Genomic studies [1] of the Apicomplexa parasite Plasmodium falciparum - the causative agent of life-threatening malaria - have confirmed earlier observations that this parasite lacks metabolic pathways for de novo synthesis of purines, and hence that purine salvage is essential for their survival. Inhibitors that block recycling of purines should therefore form a viable basis for novel malarial therapeutics. Purine nucleoside phosphorylase (PNP) in Plasmodium forms a key enzyme in the recycling of predominantly host-derived purines, catalysing the phosphorolysis of inosine to produce the major purine precur- 
sor for the salvage pathway, hypoxanthine, and ribose-1phosphate (Figure 1a). PNP also catalyses phosphorolysis of methylthioinosine, and hence is believed to play an important role in the recycling of this purine from the polyamine biosynthesis pathway [2]. Although unlikely to be of direct biological relevance, arsenate can replace phosphate in this reaction generating ribose-1-arsenate, which is then rapidly and irreversibly hydrolysed to ribose and arsenate (Figure 1b). This alternative reaction is of interest to dissect the mechanistic details of PNP and has contributed to inhibitor development. Kinetic isotope (KIE) studies have been used to study the PNP mechanism in detail $[3,4]$ and indicate that catalysis proceeds via a classic SN1 nucleophilic substitution reaction in which, at the transition state, the ribitol ring forms an oxocarbenium ion (Figure 1a). The derived conformation of this ring has been exploited in the design and synthesis of tight-binding and specific PNP inhibitors such as Immucillin-H (ImmH) (Figure 1c). ImmH is believed to mimic the transition-state formed during this reaction, and binds to the human form of the enzyme (hPNP) with higher affinity $\left(\mathrm{K}_{\mathrm{d}}=56 \mathrm{pM}\right)$ than do either the reaction substrate inosine $\left(K_{M}=40 \mu \mathrm{M}\right)$ or product hypoxanthine $\left(K_{M}=10\right.$ $\mu \mathrm{M})$. Binding of ImmH to PNP has been studied in much detail and crystal structures of its complexes with a range of PNP enzymes including bovine PNP (bPNP, together with $\left.\mathrm{PO}_{4},[5]\right)$ and Plasmodium falciparum PNP (PfPNP, together with $\left.\mathrm{SO}_{4},[6]\right)$ have been reported to resolutions of $1.5 \AA$ and $2.2 \AA$ respectively. In vivo genetic knock-out studies have recently confirmed PfPNP as the molecular target for the anti-parasiticidal activity of this family of compounds [7].

Crystal structures and sequence homology studies have distinguished two broad families of PNP enzymes: those that form trimers (such as the mammalian PNPs, Type 2) and those that are hexameric (such as E.coli PNP (EcPNP), Type 1). These groupings correlate with functional differences in that the hexameric enzymes have wider substrate specificity, in particular the ability to use 6-aminopurine a) phosphorolysis

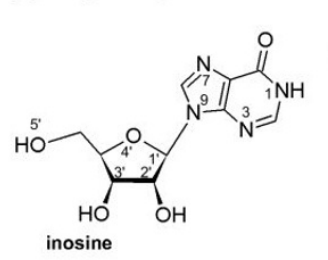

b) arsenolysis

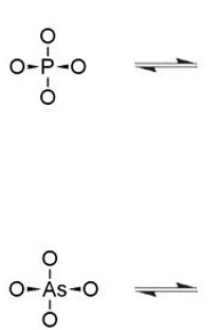

PNP transition state
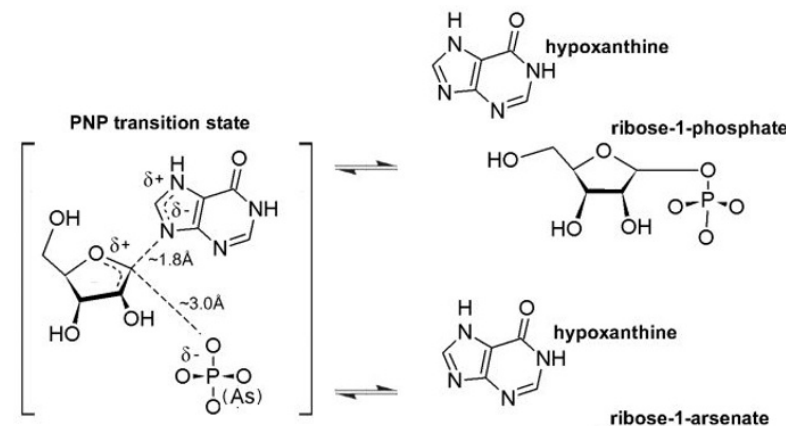

hypoxanthine



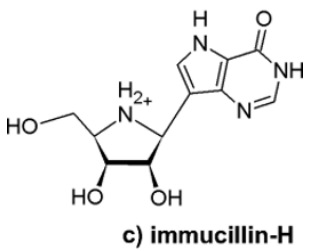

Figure I

Catalysis by PNP. Schematic diagrams showing the chemical reactions catalysed by PNP: (a) phosphorolysis, and (b) arsenolysis. These diagrams are based on arsenolytic/hydrolysis transition-state structures of PNPs [I7,3,4]. Note protonation at N7, leading to formation of a positive charge, and glycosidic-bond cleavage resulting in formation of a ribo-oxocarbenium ion and negative charge in the purine ring at the transition state. This process occurs prior to nucleophilic attack as the catalytic reaction of PNP follows a SNI type mechanism. (c) shows the transition state mimc inhibitor, Immucillin $\mathrm{H}$ (ImmH). 
nucleosides as substrates. Using phylogenetic studies [8] showed that the Plasmodium PNPs are outliers with equal genetic distance between PNPs and uridine phosphorylases. Crystallographic studies of PfPNP [6,9] have shown it forms a hexameric assembly suggesting its closer alignment with the Type 1 PNP group, although functionally it has been noted that, unlike other hexameric PNPs, adenosine is not a substrate for PfPNP. These distinguishing features suggest selectivity for PfPNP, in preference to its mammalian homologues, should be achievable in the development of novel anti-malarials. However, the generality of these distinguishing PNP features across Plasmodium parasite species remains unclear. A recent crystal structure of PNP from the closely related simian parasite Plasmodium knowlesi (PkPNP) has also been determined (entry 2b94 in the Protein Data Bank (PDB)) and, although forming a similar hexameric arrangement, the arrangement of the subunits differs from PfPNP. This largely arises as three loop regions at the subunit interface have been traced to different conformations. In addition, the arrangement of residues in the substrate binding pocket - which does not contain substrate and adjoins one of the loops and the subunit interface - differs significantly in the PkPNP structure relative to PfPNP. It is therefore unclear whether PfPNP represents an archetypal or unique member of the Plasmodium PNP enzyme family.

In this study we have therefore extended studies of Plasmodium PNPs, firstly through a structural analysis of PNP from the second most prevalent human-specific malaria parasite, Plasmodium vivax (PvPNP). Malaria due to Plasmodium vivax infection accounts for up to $40 \%$ of the annual incidence of the disease [10] and, although generally less severe, causes considerable morbidity. A genomic sequence for Plasmodium vivax has recently become available [11]. P $v$ PNP and PfPNP share $81 \%$ amino acid sequence identity. Secondly, although the enzyme mechanism involving generation of a ribitol oxocarbenium ion is believed to be general for all PNPs, the identity, contribution and movements of amino acids within the active site during catalysis differs between various forms of PNP. To further mechanistic understanding of the unusual group of Plasmodium PNPs, we have also determined the crystal structure of the arsenolytic complex of PfPNP with inosine which is found to contain a mixture of product and reactant components from the reaction. Parallel kinetic and binding studies are further used to pinpoint Plasmodium-specific features of PNP.

\section{Results \\ Crystal structure of PvPNP \\ Overall structure}

The P $v$ PNP crystal structure was refined against 1.85 A resolution data (summarised in Table 1) and the model contains all of the protein residues with the exception of the disordered active site loop (residues 212-224). The R32 crystals displayed considerable anisotropy in the distribution of their diffraction intensities leading to the rejection of many higher resolution reflections during processing. Nonetheless, about $70 \%$ of the processed reflections have intensities with I/(sigma I) greater than 3 in the highest resolution shell, hence the structure has been refined against all available data to $1.85 \AA$. The crystallographic asymmetric unit contains a monomer of $P v$ PNP which adopts the familiar single-domain fold topology described previously for hexameric PNPs from other species (e.g. $[6,9,12,13])$. Each PvPNP monomer is comprised of a 10-stranded $\beta$-sheet core, which forms the base of the catalytic site, and eight $\alpha$-helices, which are involved in subunit contacts (labelled in Figure 2). The total number and position of secondary structure elements in PvPNP are comparable to those in PfPNP, although small variations in the number of $\beta$-strands and $\alpha$-helices are evident due to different assignments when comparing PfPNP structures $([9,6]$ and the PfPNP solved in this study). An extra $\alpha$-helix in the ordered active site loop of PfPNP is not observed in the PvPNP structure, but may be formed when the P $v$ PNP active site loop is structured.

The nucleoside binding site of the PvPNP enzyme in this structure is observed to be empty although there is electron density consistent with three bound water molecules within the substrate binding site. Additionally, an anion presumably sulphate from the crystallisation buffer which contained $0.2 \mathrm{M} \mathrm{LiSO}_{4}$ - was identified and this anion occupies the phosphate binding pocket of the enzyme. Its position is consistent with sulphate and phosphate groups observed in other PNP structures including PfPNP $[6,9]$.

When the R32 symmetry operators are applied, $P v$ PNP is seen to form a hexameric assembly that matches the arrangements previously reported in crystal structures of PfPNP [6,9] and EcPNP [14]. Presumed to be the biologically-relevant structure, this hexamer is a disc-shaped single-layer with an overall diameter of about $100 \AA$ and a thickness corresponding to the width of a monomer (approximately $50 \AA$ ) with an empty central channel of diameter $20 \AA$. The hexameric PvPNP appears to be assembled from a trimer of dimers, where the dimer pairs are related by a crystallographic three-fold symmetry axis running through the central channel, corresponding to the view in Figure 2. The two monomers in each pair of dimers lie anti-parallel and are related by a crystallographic two-fold axis perpendicular to the major three-fold axis, with the two active sites lying $22 \AA$ distant from each other. 
Table I: Summary of crystallographic data.

\begin{tabular}{|c|c|c|}
\hline & PvPNP & PfPNP hypoxanthine-ribose- $\mathrm{AsO}_{4}$ complex \\
\hline PDB code & $\underline{3 E M V}$ & $\underline{3 E N Z}$ \\
\hline Space group & $\mathrm{R} 32$ & 14,22 \\
\hline \multirow[t]{4}{*}{ Unit cell } & $a=b=94.8 \AA$ & $a=b=177.8 \AA, c=253.9 \AA$ \\
\hline & $c=121.3 \AA$ & $\alpha=\beta=\gamma=90.0^{\circ}$ \\
\hline & $\alpha=\beta=90.0^{\circ}$ & \\
\hline & $\gamma=120.0^{\circ}$ & \\
\hline Resolution range $(\AA)$ & $33.98-1.85(1.92-1.85)$ & $30.94-2.03(2.10-2.03)$ \\
\hline Unique reflections & $14,619(875)$ & $129,248(12,783)$ \\
\hline Completeness (\%) & $80.5(49.0)$ & $99.4(99.8)$ \\
\hline Redundancy & $6.0(4.4)$ & $10.7(8.0)$ \\
\hline$|/ \sigma|$ & $17.6(4.3)$ & $18.5(2.7)$ \\
\hline $\mathrm{R}_{\text {merge }}$ & $0.095(0.234)$ & $0.106(0.556)$ \\
\hline Solvent content & $33.5 \%$ & $58.9 \%$ \\
\hline \multicolumn{3}{|l|}{ Refined model } \\
\hline No. Protein chains & I & 6 \\
\hline No. Protein atoms & 1921 & 11268 \\
\hline No. Other atoms (ligand, organic solvent and water) & 80 & 779 \\
\hline No. TLS groups & I & 30 \\
\hline $\mathrm{R}_{\text {fact }}$ & 0.193 & 0.160 \\
\hline Rfree & 0.233 & 0.193 \\
\hline FOM & 0.852 & 0.891 \\
\hline Mean $\mathrm{B}_{\text {fac }}$ protein $\left(\AA^{2}\right)$ & 25.7 & 16.1 \\
\hline Mean $\mathrm{B}_{f a c}$ other atoms $\left(\AA^{2}\right)$ & 29.6 & 28.8 \\
\hline rms bonds $(\AA)$ & 0.012 & 0.016 \\
\hline rms angles $\left({ }^{\circ}\right)$ & 1.611 & 1.396 \\
\hline
\end{tabular}

Table shows processing and refinement statistics for the diffraction data and models from both crystal forms. The numbers in parentheses refer to data from the highest resolution shell. 

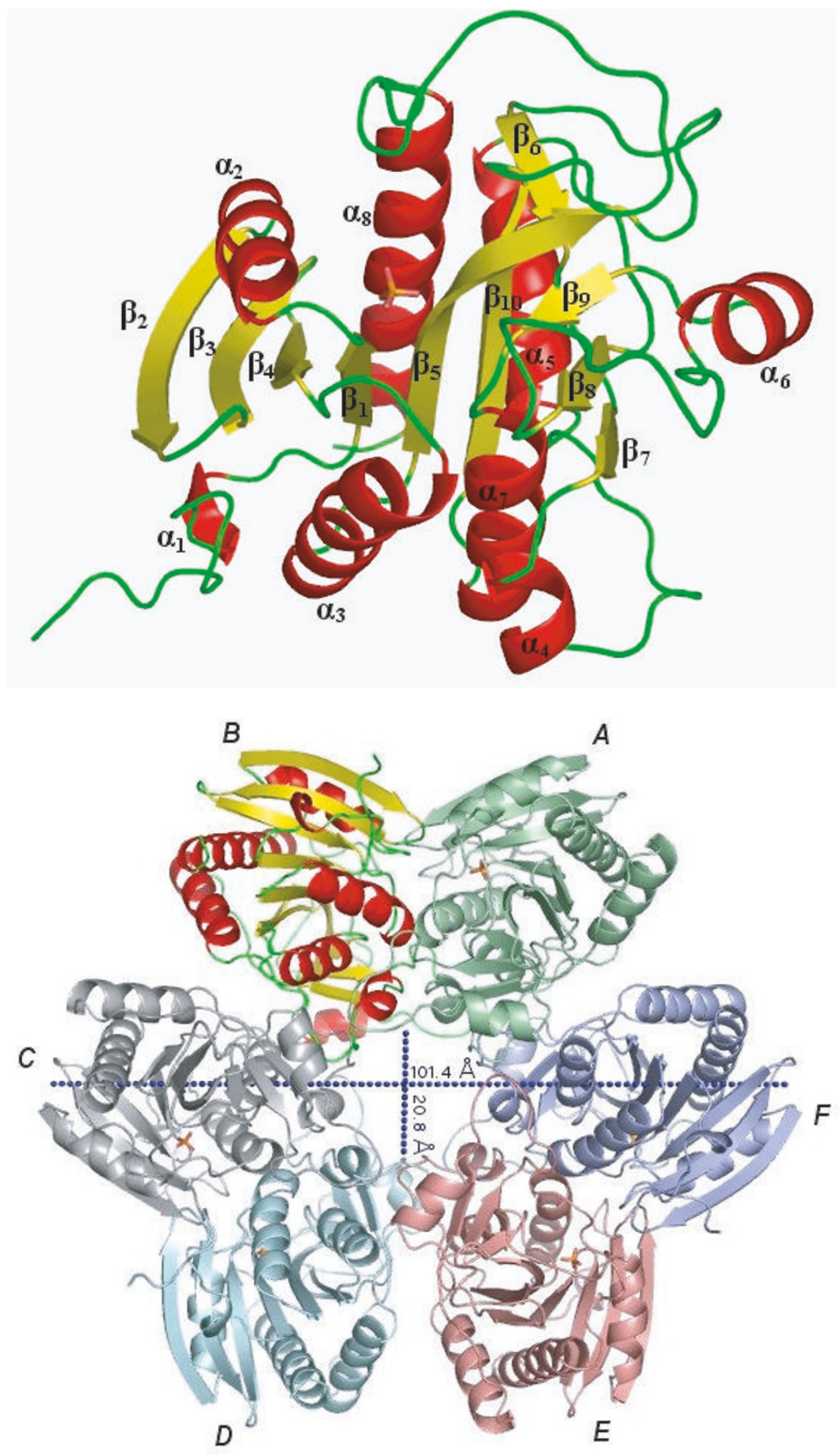

Figure 2

Overall structure of PvPNP. The ribbon diagram shows a monomer of PvPNP with the secondary structure elements labelled. The bottom panel shows the assembled hexamer with each subunit in a different colour, viewed perpendicular to the three-fold axis. 
Inter-subunit contacts in $P v$ PNP are comparable to those reported in the previous PfPNP-inosine structure [9] but different from those observed in the PkPNP structure [PDB:2B94]. The loop (residues 159-170) that connects $\beta_{8}$ and $\alpha_{6}$ is particularly evident in its contribution to the subunit interactions. This loop is adjacent to the central channel and forms contacts both with the neighbouring subunit in each dimer pair - such as A and B - and also extends to a monomer of the adjacent dimer - such as A and $\mathrm{C}$. In the former case, a distinctive hydrogen bond is formed between Tyr 162 of chain A and Glu 78 of chain B, which has also been described for PfPNP [9] and PNP from Thermus thermophilus (TtPNP) [15]. The length of this loop differs between hexameric PNP enzymes from different species. Relative to PkPNP and TtPNP, the $P v$ and Pf enzymes have loops extending about 11 A further from the core, whereas in EcPNP the equivalent loop extends about $6 \AA$. In the PfPNP-inosine structure, Schnick et al. [9] showed that this loop also contacts the ligand and proposed that this elongated loop plays an important role for not only quaternary structure formation but also in determining accessibility to and the conformation of the active site cavity. With no substrate bound in the current structure it is not possible to confirm this is also the case for the $P v$ PNP enzyme.

\section{The active site of PvPNP}

In common with $P f \mathrm{PNP}$, the phosphate/sulphate binding site of $P v$ PNP (Figure 3 ) is formed mainly by two arginine residues (Arg 89 and Arg 46' from the neighbouring monomer), a backbone interaction with Gly 24, and both backbone and side chain interactions with Ser 91 . Another arginine, residue 27, also lies in this region, but points away from the bound sulphate in the PvPNP structure. This is similar to the arrangement in PfPNP with sulphate bound [9] but differs from PfPNP with arsenate bound (see below). Overall, the $\mathrm{PO}_{4} / \mathrm{SO}_{4}$ binding pocket in $P v$ PNP is essentially identical to that previously described for PfPNP.

Although neither base nor sugar are bound in the nucleoside binding site of the $\mathrm{P} v \mathrm{PNP}-\mathrm{SO}_{4}$ structure, these sites can be readily identified by overlaying the hypoxanthine and ribose sugar molecules from the PfPNP-hypoxanthine-ribose- $\mathrm{AsO}_{4}$ structure (see next section). This shows that the base would be primarily accommodated via $\pi$ stacking of Tyr 161 and Pro 210, and also Trp 213 when the active site loop (207-225) is ordered. Other significant contributors include Ser 92, Val 182, Cys 93 and Gly 94. Asp 207, which has been proposed to play an important role in stabilisation of the transition state complex $[6,16]$ is also present in the PvPNP purine-binding site, although in a conformation typical of an empty binding site [9]. The composition and structure of the base binding pocket in $P v$ PNP is essentially identical to that described for PfPNP, whereas there are substantial differences when compared to this region in the PkPNP structure. Although the identity of the key residues is unaltered in PkPNP, the altered conformation of the 159-170 loop results in a substantial (4 $\AA$ ) displacement of Tyr 157 which, along with Tyr158 (equivalent to Tyr 161 and Tyr 162 in PvPNP), now occupies the space in which the base normally binds. This leads to considerable distortion of the base binding pocket.

Similarly, the sugar binding site of $P v$ PNP is virtually identical to that of PfPNP. By analogy with the PfPNP complex, the sugar $\mathrm{O} 2$ ' and $\mathrm{O}^{\prime}$ ' hydroxyl groups are expected to interact with the side chains of Arg 89 and Glu 185. His $8^{\prime}$ from the neighbouring monomer is in a position to bind the ribose O5' hydroxyl group and Ser 92 is positioned to hydrogen bond to the ribosyl ring $\mathrm{O}^{4}$. Other residues such as Tyr 161, Met 184 and Val 67 also line the cavity in both structures. By contrast, although many of the above interactions are also possible in the PkPNP structure, the altered conformation reported for the 159170 region once again leads to changes in the sugar binding pocket, with His 8 ' considerably displaced and its equivalent position now occupied by Lys 164 . Tyr 160 , as discussed above, is present but displaced by about $4 \AA$ and there is no direct equivalent to Val 67, although its role may be partially replicated by Ala 66 .

\section{Crystal structure of PfPNP complexed with hypoxanthine, ribose and arsenate}

This structure was obtained by co-crystallising PfPNP with inosine and arsenate to produce a PfPNP, hypoxanthine, ribose and arsenate complex (PfPNP-HRA) (see below). The asymmetric unit consists of one hexamer of PfPNP in which there are six very similar, but not identical, subunits. The crystal packing differs from previously reported structures for PfPNP [6,9] although the arrangement of monomers is very similar. All monomers in the hexamer have essentially the same overall secondary structure (root mean square deviations (rmsd) of equivalent Cas between monomers range from $0.16-0.40 \AA$ ) with the exception of only one area. This is in the active site loop (206-224), part of which forms a short helix in four monomers $(\mathrm{A}, \mathrm{B}, \mathrm{D}$ and $\mathrm{E})$ whereas this is not present in the remaining two chains ( $\mathrm{C}$ and $\mathrm{F})$.

\section{The active site}

Although inosine and arsenic acid were included in the crystallisation mixture with PfPNP, the resulting electron density was inconsistent with inosine being present in the active site. The PNP-catalysed arsenolysis reaction is similar to phosphorolysis, however the ribose-1-arsenate product is unstable and is rapidly hydrolysed into ribose and arsenate [17] as shown in Figure 1. 

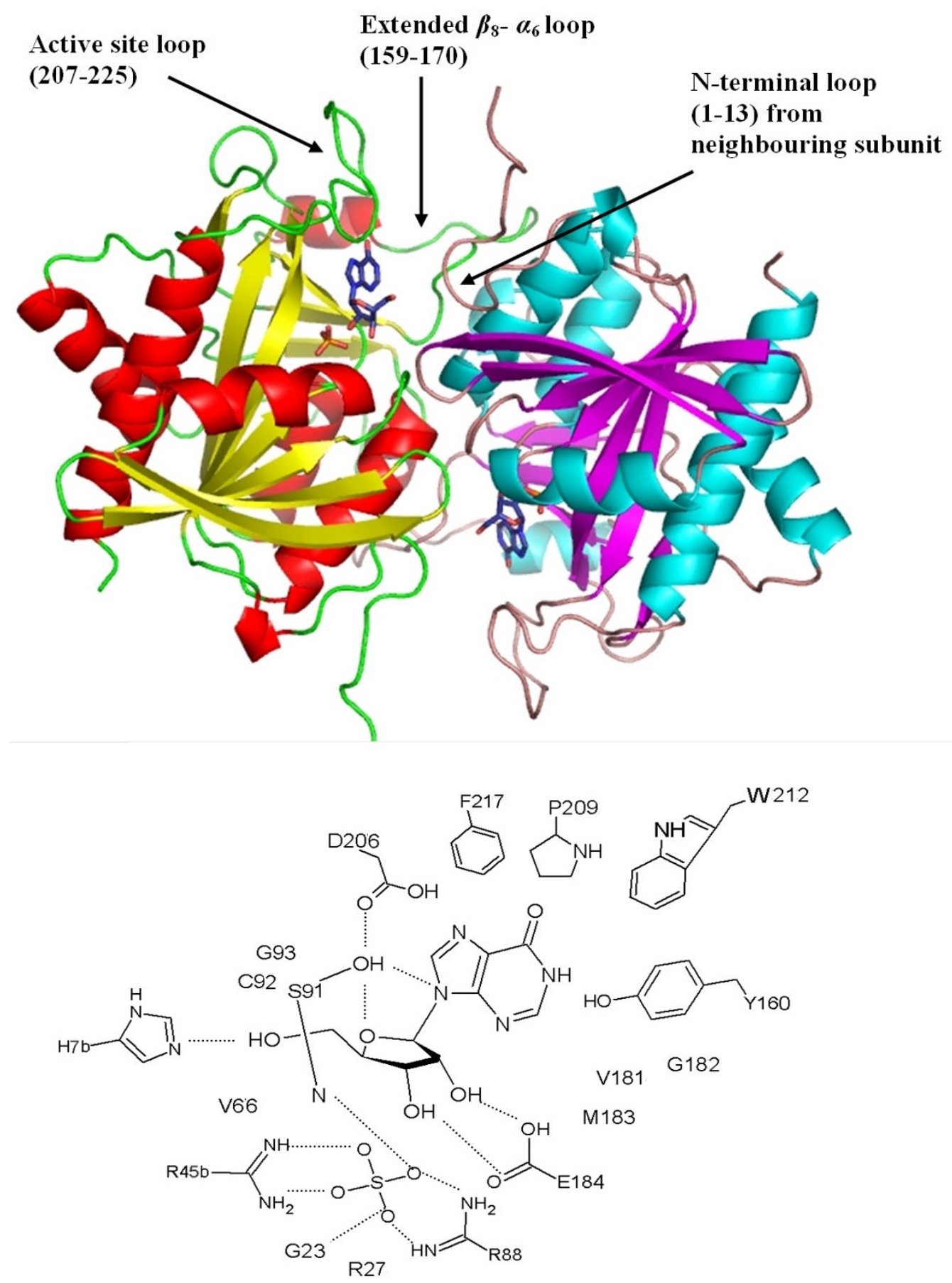

\section{Figure 3}

The active site of the PrPNP. The top panel illustrates the dimer pair and their binding sites with the bound sulphate anions (from the crystal structure) and the inosine substrates (modelled into the PvPNP structure based on superimposition of the PfPNP-inosine structure, PDB:2BSX). The bottom schematic diagram shows possible key residues of the PvPNP active site (numbered according to the system previously used for PfPNP) with hydrogen bonds as dashed lines. Most residues are from the parent monomer, while those labelled 'b' are from the neighbouring subunit across the dimeric surface. 
In accordance with the shapes, sizes and positions of electron density observed within the active site, the products hypoxanthine base, ribose sugar and arsenate anion $\left(\mathrm{AsO}_{4}\right)$ - formed from the irreversible arsenolysis of inosine followed by hydrolysis of the ribose-1-arsenate - were modelled in to the observed electron density map. These proved to be a good match for the density and were wellbehaved in subsequent refinement. Based on the known sequential mechanism for release of the products [18], the inclusion of the arsenate group correlates with the presence of the ribose sugar which consecutively matches the presence of hypoxanthine. These product and reactant molecules are assumed to be accommodated within the active site in a similar way to enzyme intermediates in accordance with the observed catalytic conformation of the enzyme, including a closed active site loop with the characteristic helical segment and the side-chain conformation of Arg 27 (see below).

The binding pocket is clearly defined in this structure (Figure 4). In essence, the composition of the pocket is identical to that previously described in the PfPNP structures $[6,9]$. However, there are important differences in the conformations of several key catalytic residues.

\section{Arsenate binding site}

In all subunits, the arsenate moiety is stabilised mainly by two arginine side chains (Arg 88 and Arg 45' of the neighbouring subunit) and further hydrogen bonds are formed with donors from the hydroxyl group of Ser 91 and the backbone amino groups of Gly 23 and Ser 91. These interactions are similar to those observed for the bound sulphate in the PfPNP-SO ${ }_{4}$ [9] and the PfPNP-ImmH [6] structures. However the participation of the Arg 27 residue in the anion binding pocket, where its guanidinium side chain forms charged hydrogen bonds with the arsenate molecule, has not been observed in structures with sulphate bound $[16,19]$. Despite the inclusion of arsenic acid, this complex was crystallised under conditions heavily buffered to neutral $\mathrm{pH}$ (4 M sodium formate) and hence the ionisation state of the arsenate is expected to be the same as for sulphate (both are dianions at this $\mathrm{pH}$, believed to be the active form in catalysis [20]). Similarly, as both crystal structures have been determined at neutral $\mathrm{pH}$ no alterations in the ionisation states, and hence overall conformation, of the three arginine residues that dominate the arsenate/phosphate binding site would be expected. In the PfPNP-HRA complex, in five of the subunits the Arg 27 side chain points toward the active site ('closed' conformation, average torsion angle $\chi_{1}=163^{\circ}$ and $\chi_{2}=175^{\circ}$ ) and forms hydrogen bonds with oxygen atoms of the arsenate ion. This conformation appears to increase ordering of the anion and leaves little unoccupied space in the binding pocket. By contrast, in chain $\mathrm{F}$ this arginine is in an 'open' conformation with its side chain pointing away from the active site (torsion angle $\chi_{1}$ $=178^{\circ}$ and $\chi_{2}=75^{\circ}$ ) and hence does not interact with the arsenate ion.

Nucleoside (ribose sugar and hypoxanthine) binding site

All six binding pockets in the hexamer bind both ribose and hypoxanthine in a similar conformation with equivalent hydrogen-bond interactions formed to residues in each active site (Figure 4). The ordered active site loop brings Pro 209, Trp 212 and Phe 217 into the hydrophobic pocket, resulting in the hypoxanthine being oriented by $\pi$-stacking and van der Waals interactions. Similar to the PfPNP-Imm complex, the carboxylate group of the Asp 206 side chain interacts directly with N7 of the hypoxanthine, and has been proposed to be the general acid/ base for protonation of $\mathrm{N} 7$ of the substrate in the transition state $[14,6,16]$. The conformation of this flexible Asp side chain differs from that observed in the PfPNP-SO and PfPNP-ino structures where Asp 206 points away from the active site and forms hydrogen bonds to the hydroxyl group of Ser 91 .

Also of interest is a bound water molecule close to $\mathrm{O} 6$ of the purine base, which is present in all subunits with Arg 27 in the 'closed' conformation. This water molecule acts as a bridge linking Trp $212\left(\mathrm{~N}_{\varepsilon 1}\right)$ (a residue from the active site loop), Asp $206\left(\mathrm{O}_{\delta 1}\right)$ and $\mathrm{O} 6$ via a hydrogen-bond network (Figure 4), and hence may play a role in catalysis or stabilisation of the transition state. In contrast, this water molecule is not observed in chain $\mathrm{F}$, consistent with a state in which the active site loop is destabilised.

The ribose sugar binds in between the arsenate anion and the hypoxanthine base with its oxygen atoms (both hydroxyl oxygens and $\mathrm{O} 4$ ') fully engaged with hydrogen bonds formed with several residues (Figure 4). The interactions in this region are not significantly different from those previously described in the PfPNP-ino [9] and the PfPNP-ImmH [6] structures. There appear to be no conformational alterations associated with binding of the ribose sugar, consistent with the observation in the EcPNP structure [16].

In summary, the hexameric structure of the PfPNP complex appears to contain two states of the enzyme. In five of the subunits (chains A - E) Arg 27 is in a 'closed' conformation and the $\alpha_{8}$ helix is formed within the active site loop. In the remaining subunit (chain F) Arg 27 is in an 'open' conformation and the bound water close to $\mathrm{O} 6$ is absent.

\section{Enzyme Kinetics}

Kinetic data for PfPNP and PvPNP are summarised and compared with existing published data $[8,21-25]$ in Additional File 1 . These data are similar to those previously 

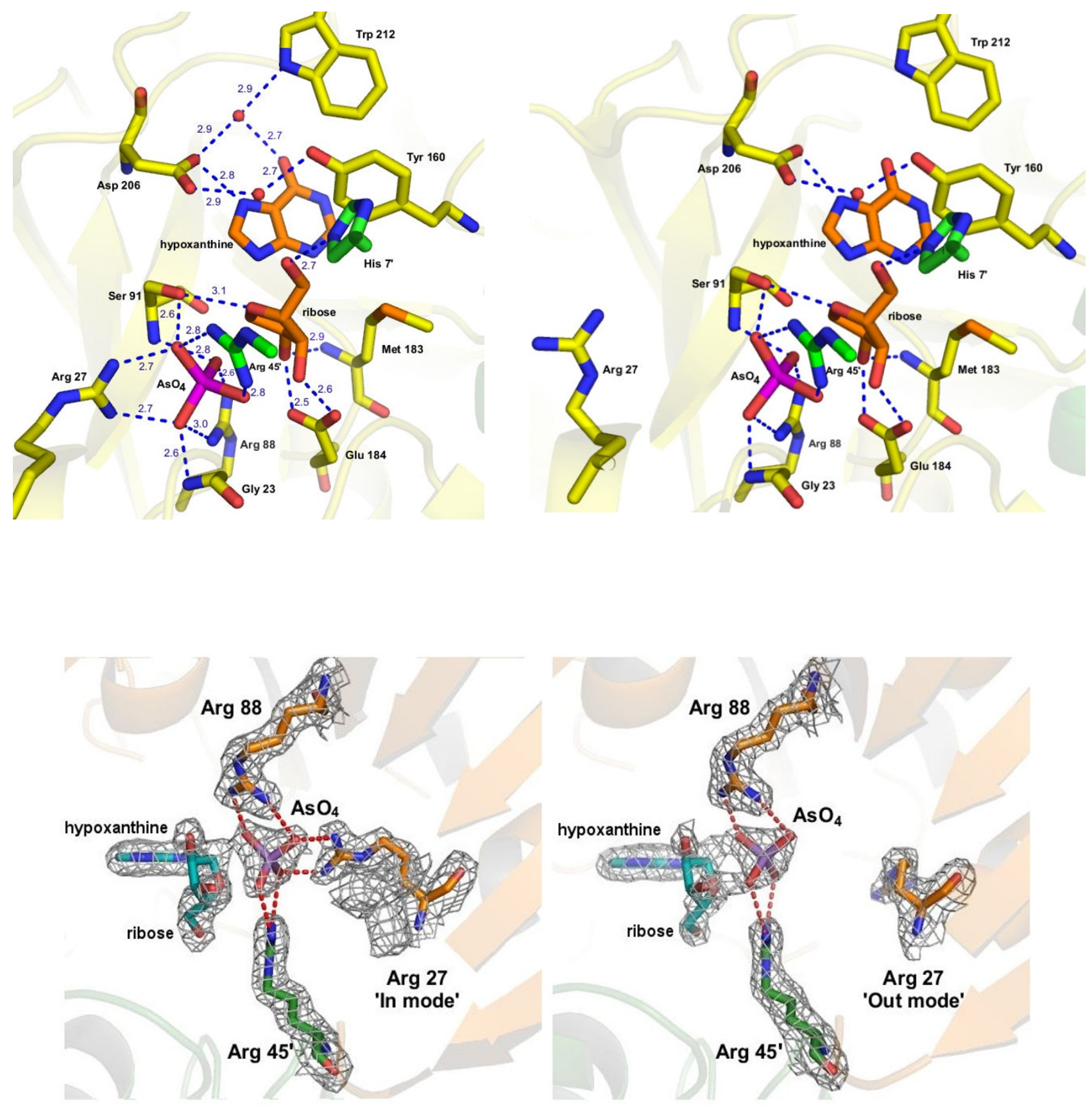

\section{Figure 4}

Structure of Pf PNP-HRA complex. (a) active site of typical subunit (b) active site of subunit F (c) corresponding electron density $\left(2 \mathrm{~F}_{\text {obs }}-\mathrm{F}_{\text {calc }}\right.$, contoured at I sigma) for the 'in' and 'out' modes of Arg 27 respectively. Hydrogen bonds are shown as blue dashed lines with distances in Ångstroms, and bound water molecules are shown as red spheres. 
reported for $P f \mathrm{PNP}[8,22]$ with the exception that $P v \mathrm{PNP}$, unusually, appears to be activated by the 2'-deoxyinosine substrate as discussed below. This increase in rate was consistently observed with both different batches of $P v$ PNP and altered concentrations of the linked enzyme, xanthine oxidase, and hence appears to be an inherent characteristic of $P v$ PNP with 2'-deoxyinosine. The Plasmodium PNPs are seen to display slightly better catalytic efficiency for guanosine than inosine, consistent with previous reports.

\section{Discussion}

Reconciliation of structures derived from the arsenolytic reaction of PNP with the mechanism of phosphorolysis is complicated primarily by the spontaneous breakdown of the ribose-1-arsenate product. This leads, in this study, to a complex that contains a mixture of products (hypoxanthine), a (non-enzymatic) degradation product of the real product ribose-1-arsenate (ribose) and reactants (arsenate). The relative placement of these groups within the active site differs from previous structures of PNP complexes containing products, reactants or inhibitors.

Two different forms of the active site are observed in the PfPNP-HRA complex. These are distinguished by the conformation of the active site loop, and the positioning of the side chain of Arg 27. Conformational changes in the active site loop have previously been reported as a distinguishing structural feature between the ground-state (e.g. PfPNP-SO4 or PfPNP-ino, loop disordered) and catalyticstate (PfPNP-Imm, loop forms a short helix) of PfPNP $[6,9]$. In the PfPNP-HRA complex, although all active sites are occupied, in four subunits $(\mathrm{A}, \mathrm{B}, \mathrm{D}, \mathrm{E})$ the active site loop is ordered - indicative of a catalytically-active form whereas its disorder in subunits $\mathrm{C}$ and $\mathrm{F}$ may reflect the start of a transition in which the enzyme adopts a 'relaxed state' to release the products of arsenolysis. This is supported by the conformation of the Arg 27 side chain which points away from the active site in chain $\mathrm{F}$, in contrast to Chain $\mathrm{C}$ and the other chains where this side chain participates in binding the arsenate group (Figure 4). This has not been observed previously in PfPNP-Imm structures (co-crystallised with $\mathrm{SO}_{4}$, [6]) although conformational alteration of an equivalent arginine side chain has previously been noted to correlate with enzymatic activity in EcPNP where the 'open' position of the arginine side chain has been correlated with the enzyme in a non-catalytic state $[16,19]$. In the EcPNP enzyme the two conformations were also proposed as an explanation for the two different phosphate affinities observed [16].

Another feature of the PfPNP-HRA active site that provides some insight into the mechanistic state reflected by the complex is the binding of Asp 206 directly to the N7 of the base, an arrangement consistent with its proposed role as the general acid/base for protonation of N7 of the substrate in the transition state $[14,6,9,16]$. It has been suggested that this carboxylate-base interaction is exclusive to the catalytic state structure, and is unlikely to play an important role in initial substrate binding [9]. By this criterion the conformation of Asp 206 observed in the present structure implies that this structure of PfPNP represents the enzyme in a catalytic state. This is further supported by the observation of a bound water molecule close to $\mathrm{O} 6$ of the purine base, which is present in all subunits with Arg 27 in the 'closed' conformation. This is similar to the reported structure for $E c P N P$ where the presence and absence of the equivalent water molecule correlates with the two states for the active site loop, and has lead to the suggestion that the water molecule might act as a lubricant for the folding and unfolding of the helix in the active site loop [16].

Together, these indicators are all consistent with five subunits of the PfPNP-HRA complex representing an active conformation of the enzyme in an intermediate state structure. In contrast, the remaining subunit (chain $\mathrm{F}$ ) may represent the structure of the enzyme in a non intermediate state or, speculatively, a state prior to release of the products.

\section{Mechanism of Plasmodium PNPs}

The catalytic mechanism of PNP enzymes has been dissected in detail in many previous studies. Nevertheless, the PfPNP-HRA complex in this study provides an interesting addition to the many crystallographic observations that support a mechanism elucidated primarily by KIE studies. Firstly, arsenate is chemically and physically more similar to phosphate than is sulphate, which has been used extensively in many of the previous PNP crystal structures. Secondly, to form the oxocarbenium ion transition state the $\mathrm{C} 1$ ' atom of the ribose ring is required to move away from the base by about $0.3-0.4 \AA$ [17] and then further towards the phosphate ion to form partial bonds to both the purine and the phosphate, a position known as the point of atomic symmetry in the reaction coordinate of PNP, and which is energetically similar to the complex with bound products [5]. At this point the C1' atom lies equidistant between the N9 of the base and the incoming phosphate/arsenate oxygen nucleophile. This condition is close to being satisfied in the crystal structure of the PfPNP-HRA complex, with average separations of As-O1 $\cup \mathrm{C} 1$ ' of $2.4 \AA$, and $\mathrm{C} 11^{\prime} \cup \mathrm{N} 9$ of $2.7 \AA$. By contrast, the equivalent distances in the PfPNP-ImmH complex are $3.4 \AA$ and $1.6 \AA$ respectively, and $3.6 \AA$ and $1.5 \AA$ in the PfPNP-ino and PfPNP-SO ${ }_{4}$ complexes (see Figure 5, which is similar to previous figures such as in $[5,26,27])$. The ribose C3' atom is seen to adopt an exo conformation, consistent with the observation for the conformation of iminoribitol group of immucillin $\mathrm{H}$ in 

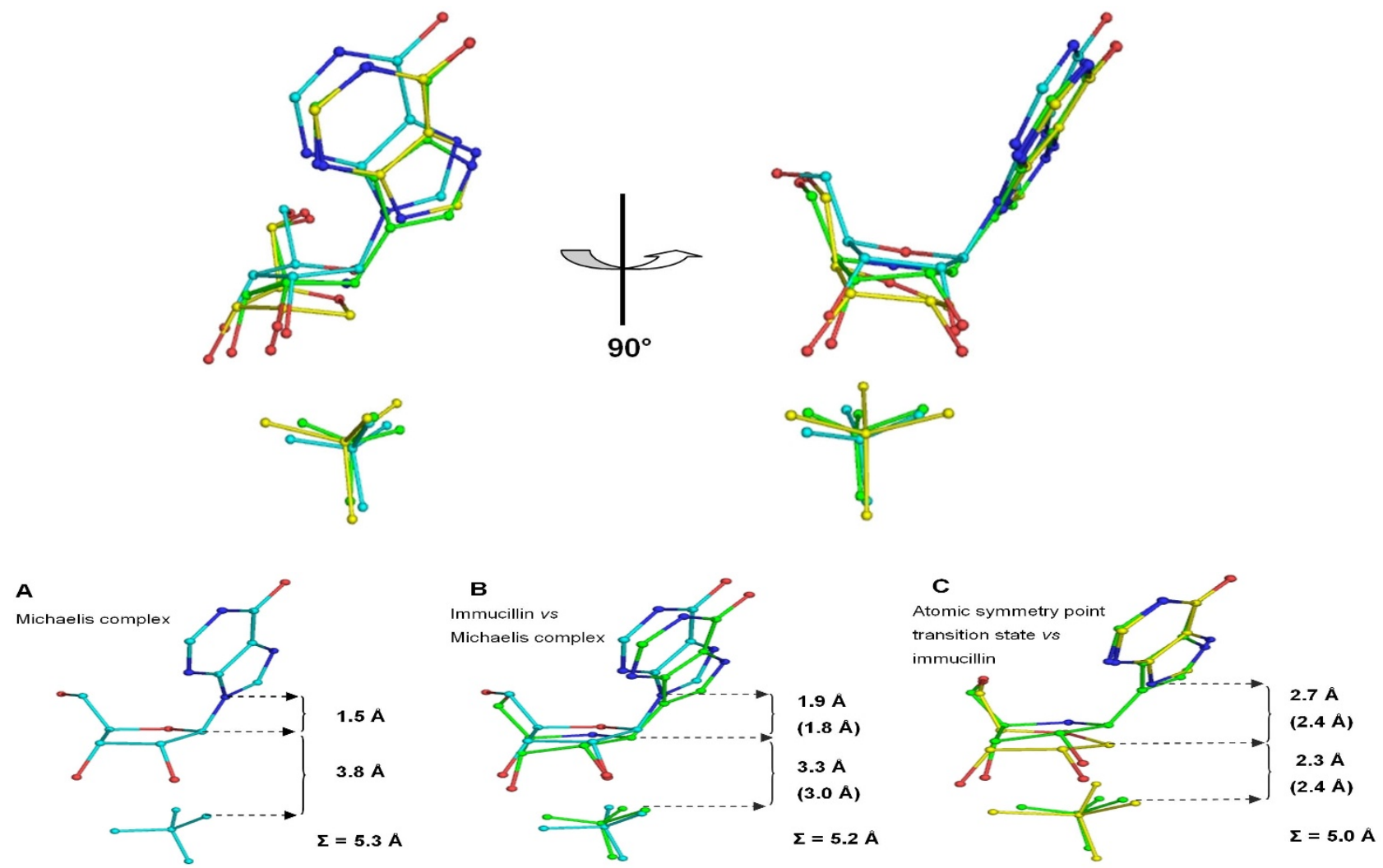

\section{Figure 5}

Movement of the $\mathrm{CI}$ ' of the ribose ring throughout catalysis by pPNP. Top figure shows two perpendicular views of an overlay of the ribose and surrounding atoms in PfPNP-inosine/PfPNP-SO 4 (Michaelis complex, green), PfPNP-ImmH (transition state, purple) and PfPNP-hypoxanthine-ribose-As $\mathrm{O}_{4}$ complex (post-transition state, yellow) structures. Bottom panel show the distances between $\mathrm{Cl}^{\prime}-\mathrm{N} 9$ and $\mathrm{Cl}^{\prime}$-nucleophilic oxygen when comparing ligands in different states with the sum of the reaction coordinate distance $(\Sigma)$ shown. The numbers in brackets are the values proposed by KIE calculations between the Michaelis complex and transition state [17] or those observed in bPNP crystal structures representing the transition state and post-transition state [5]. This figure is similar to those compiled previously for other PNP combinations such as in [5,26,27].

the PfPNP-ImmH structure (3), and has an O5'-C5'-C4'C3' dihedral angle of $174^{\circ}$. This differs from an earlier suggestion from KIE studies that a C3'-endo conformation might be adopted during catalysis (4). The proximity of the $\mathrm{C} 1$ ' atom to the nucleophile is consistent with a posttransition state arrangement. By contrast, PfPNP-ino and PfPNP-ImmH complexes are pre-transition state and (close to) transition state conformations respectively. In combination, the series of PfPNP structures shown in Figure 5 provides a neat series of snapshots illustrating clearly the movement of the $\mathrm{C} 1$ ' atom of the ribose group throughout the phosphorolysis mechanism in PfPNP.

Further examination of the association of the ligands with PfPNP also suggests the active site arrangement of the complex may be closer to an intermediate state rather than a straightforward product complex. Firstly, the $\mathrm{AsO}_{1}$ $\cup \mathrm{C} 1$ ' distance is longer and the $\mathrm{C} 1^{\prime} \cup \mathrm{N}$ 9 distance is shorter than those previously described for true product complexes of mammalian PNPs (1.5 ̊ and $3.8 \AA$, respectively [28]). Further, by comparison with PNP complexes with sulphate or phosphate, the arsenate oxygen is tilted towards the ribose ring oxygen $\left(\mathrm{AsO}_{1} \cup \mathrm{O} 4^{\prime}=3.2 \AA\right)$ in a similar arrangement to that seen in the ImmH structure $\left(\mathrm{PO}_{1} \cup \mathrm{N}^{\prime}{ }^{\prime}=3.3 \AA\right)$. This arrangement in $\mathrm{ImmH}$ is believed to reflect partial charge on the iminoribitol ring indicative of ribo-oxocarbenium ion character [5]. In addition, the $\mathrm{N} 9$ to $\mathrm{O}_{1}$ distance of the bound $\mathrm{SO}_{4}$ is $4.7 \AA$ in the PfPNP-ImmH complex, and $4.8 \AA$ in the PfPNPHRA complex $\left(\mathrm{AsO}_{4}\right)$. Closer distances in these complexes 
are generally believed to indicate more transition-like character, representative of transition state formation with significant bond order to leaving and/or attacking groups. Finally, the involvement of Arg 27 in the PfPNP-HRA complex distinguishes this conformation from that previously described for the PfPNP-ImmH transition-state complex.

Previous studies $[16,19]$ have suggested that Arg 27 not only enhances the affinity of binding of the phosphate group, but also participates in catalysis by stabilising the negative charge of the anion. However, the role of Arg 27 may be more complex. Erion et al. [20] proposed that a basic residue in this location at this position may be involved in preparation of a catalytically active anion containing nucleophilic oxygen, by demonstrating an important role for the His 86 residue found in this location in human PNP. This histidine is believed to deprotonate the anion, hence generating the required catalytically preferred ionic state and strengthening the negative charge of the bound phosphate/arsenate anion [29]. Of the three arginines within the active sites of pPNPs, since the immobile Arg 88 and Arg 45' participate in the binding site in both the ground and intermediate states, it appears that Arg 27 may fulfil a similar role to His 86 in the human enzyme, helping to stabilise a negative charge on the phosphate anion and hence leading to activation of the nucleophile in the intermediate state. In the PfPNP-HRA complex, Arg 27 forms bifurcated hydrogen bonds (average distance $2.7 \AA$ ) directly with two of the oxygens of the bound arsenate, consistent with this proposed role. This interaction is absent in the PfPNP-ImmH structure, in which the arginine side chain is turned away from the bound sulphate, as also seen in one of the subunits (F) in the PfPNP-HRA complex.

In discussion of the role of a similar arginine (Arg 24) in EcPNP, [16] proposed that its structural rearrangement is induced by a tight-binding enzyme conformation, in which a neighbouring continuous helix is broken into two parts, one of which moves in the direction of Arg 24. This helix brings Arg 217 close to Arg 24, permitting the formation of a hydrogen bond between $\operatorname{Arg} 24 \mathrm{~N}_{\varepsilon}$. and the $\operatorname{Arg}$ 217 main chain oxygen. This structural rearrangement is not the case for PfPNP; nonetheless, stabilisation of Arg 27 is still observed but must result from a different mechanism. We note that the $\mathrm{N}_{\varepsilon}$ atom of Arg 27 is instead surrounded by several water molecules forming bridging interactions between this $\mathrm{N}_{\varepsilon}$ atom and the $\mathrm{O}$ atom of Asn 219 and $\mathrm{O}_{\delta 2}$ atom of Asp 24. As phosphate and arsenate ions are similar in size and - under the crystal conditions - charged, binding of both molecules to the protein is likely to follow the same scheme.
As the composition of the active sites is identical between the PvPNP and PfPNP structures, it appears reasonable to conclude that both enzymes use the same residues and have the same catalytic mechanism. Fusing a multitude of previous mechanistic studies of other PNPs with the various Plasmodium PNP crystal structures enables a generic mechanism for the Plasmodium enzymes to be summarised (Figure 6). This differs from the mammalian (trimeric) PNP mechanism primarily in the identity and contributions of several of the key catalytic amino acids. These include the anion binding site which is formed from three arginine residues in pPNPs, whereas from one arginine and one histidine in hPNP; the proton-donating residue is Asp 206 in pPNP and Asn 243 in hPNP; residues in the ribose binding pocket also differ particularly a charged Glu 184 in pPNP relative to the hydrophobic Tyr 88 in hPNP, and in PPNP there are cavities adjacent to the O5' of the ribose and $\mathrm{N} 1$ and $\mathrm{C} 2$ of the base, which are filled by hydrophobic residues for the former and Glu 201 for the latter in hPNP (Figure 7). These accumulated differences suggest that selectivity for the Plasmodium forms should be achievable in the design of PNP inhibitors. This has already been shown with the ImmH series of inhibitors where the derivative MT-ImmH has been reported to bind to PfPNP over 100 fold tighter than to human PNP [6].

\section{Substrate activation in PvPNP}

Phosphorolysis of most nucleoside substrates by PfPNP and $P v$ PNP in this study followed the typical mechanism described by the Michaelis-Menten equation - i.e. double reciprocal plots of $1 / v$ versus $1 /[\mathrm{S}]$ produce linear relationships (data not shown). However, this was not the case for the reaction of $P v$ PNP with 2'-deoxyinosine. Although 2'deoxyinosine is unlikely to be a biologically-relevant substrate for Plasmodium PNP, it is similar to some of the developed PNP inhibitors such as 2'-deoxy immucillin-H and G. Substrate inhibition was first considered as an explanation for the non-linearity of the reciprocal plot for this substrate, but the data fitted poorly to the MichaelisMenten equation corrected for substrate inhibition $\left(v_{0}=\right.$ $V_{\max }{ }^{*}[\mathrm{~S}] /\left(K_{\mathrm{M}}+[\mathrm{S}] *\left(1+[\mathrm{S}] / K_{\mathrm{i}}\right)\right)$ (Figure 8$)$. Plots of $v_{0}$ versus $\log [\mathrm{S}]$ (data not shown) also confirmed that curvature was not symmetrically bell-shaped, which is typical for substrate inhibition cases [30].

There have been previous reports of substrate activation of the trimeric hPNP with specific nucleoside substrates [31,32]. The data for $P v$ PNP with 2'-deoxyinosine were therefore fitted to a derived substrate activation equation following the rapid equilibrium method described by [30] and showed an improved fit (Figure 8A) leading to calculation of the kinetic parameters shown in Additional File 1 . It was also noted that a reciprocal plot of $1 / v$ versus $1 /$ $[S]$ is linear over the low substrate concentration range but 

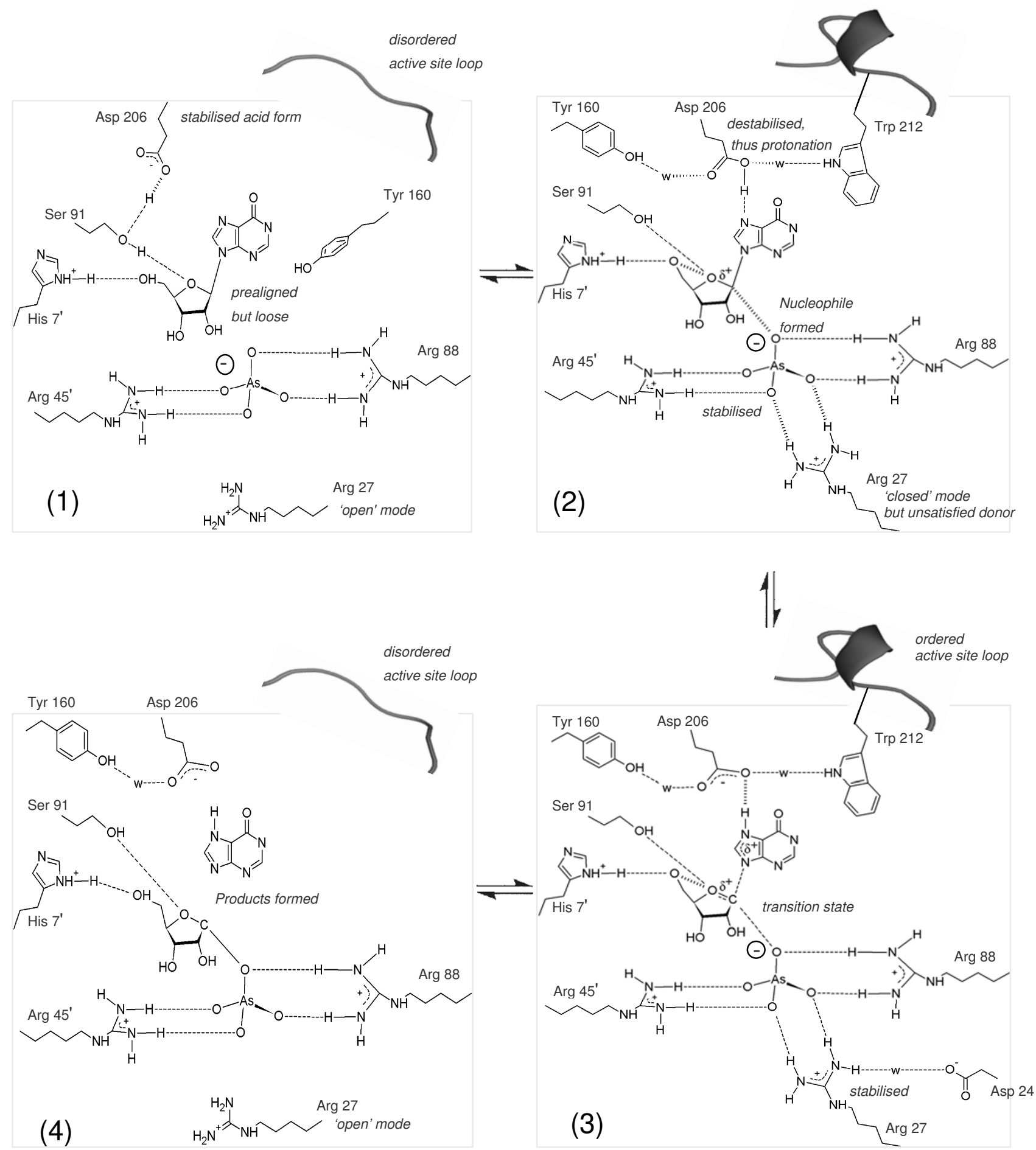

Figure 6

Catalytic mechanism of Plasmodium PNP. Schematic diagram showing the proposed generic Plasmodium PNP reaction mechanism, which is based on that initially proposed for EcPNP [19]. Note that Asp 206 must be in its acidic form prior to protonation. The dotted lines indicate electrostatic interactions, dashed lines are hydrogen bonds and ' $w$ ' indicates water molecules. Panel (I) is the binding state, (2) is the pre-catalytic state, (3) is the intermediate state, and (4) is the pre-leaving state. See text for details of each step. 

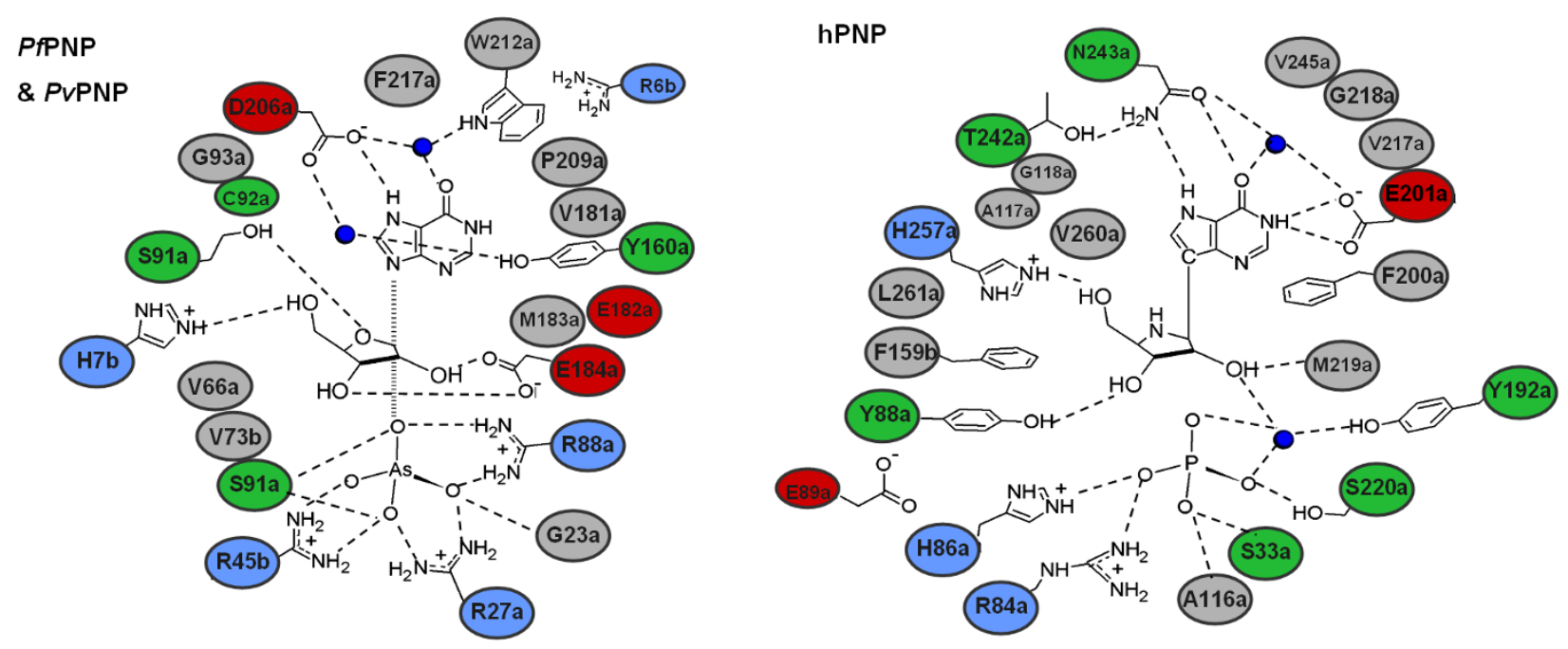

\section{Figure 7}

Substrate placement and binding pocket in the Plasmodium and human PNP enzymes. The schematic diagrams represent the human and parasite forms of the enzyme at the catalytic state. The diagram for Plasmodium parasite enzymes (left) is based on the arsenolytic-intermediate-state PfPNP and the sulphate-bound PvPNP structures, with the residues numbered according to the $P$. falciparum enzyme (+I for PvPNP), while that for human PNP (right) is based on the transition-stateanalogue complexes (PDB ID: IRR6, [6]) and refined atomic coordinates [20]. Amino acids lining the active sites are represented by spheres, coloured grey for non-polar, green for uncharged polar, blue for positively charged and red for negatively charged amino acids with bound water molecules shown as dark blue spheres. Note residues labelled 'a' are from the parent subunit, while those labelled 'b' from the neighbouring subunit in a dimer pair.

turns upwardly concave over a high substrate concentration range (Figure $8 \mathrm{~B}$ ). Frieden et al [30] noted that for substrate activation cases this curvature is normally downward, although $P v$ PNP with the 2'-deoxyinosine substrate appears to be an interesting form which has also been found in threonine dehydrase [33] and can nevertheless be classified as a substrate activation phenomenon. This distinguishes substrate activation in PvPNP with 2'-deoxyinosine from other examples of substrate activation reported for other PNP enzymes [31,32] which conform to the classical substrate activation model. Application of the Hill equation to the first portion of the PvPNP-2'deoxyinosine data confirmed the existence of positive cooperativity (Hill coefficient of 1.9, data not shown), consistent with the notion of substrate activation of $P v P N P$ by 2 -deoxyinosine, a property not shared by PfPNP despite the high overall structural similarity. However, the mechanism of substrate activation in PNP enzymes, particularly the unusual form observed here for P $v$ PNP, is not evident from the crystal structure. For the other substrates, the 2' hydroxyl group forms a hydrogen bond $(2.5 \AA)$ with Glu 184 . The absence of this interaction when 2 -deoxyinosine is used as substrate might facilitate positioning rearrangements between the ribose and phosphate, hence explain- ing the increased $k_{\text {cat }}$ rate. Nonetheless, there is no obvious explanation in the structures as to why this capacity might vary between the PfPNP and PvPNP enzymes, the former not displaying substrate activation.

It is also worth noting that despite strict conservation of the active site composition and structure between the $P f P N P$ and P PPNP enzymes small variations in their enzymatic activities are observed. These might arise through indirect effects from amino acid changes peripheral to the active site region, as has recently been described for mammalian PNPs [34,35]. In the assembled dimers of Plasmodium PNPs the amino terminus of each neighbouring subunit is located close to the adjacent subunit nucleoside binding site. This region has the greatest number of amino acid differences between the two Plasmodium enzymes, including a number of charge changes. These substitutions may induce electrostatic changes in the enzyme leading to alterations of the $\mathrm{pKa}$ values for the active site histidines (His 7 in PfPNP or His 8 in PvPNP), a mechanism that has previously been demonstrated, for example, to explain the differential activities of isoforms of human lactate dehydrogenase despite their identical active sites [36]. 

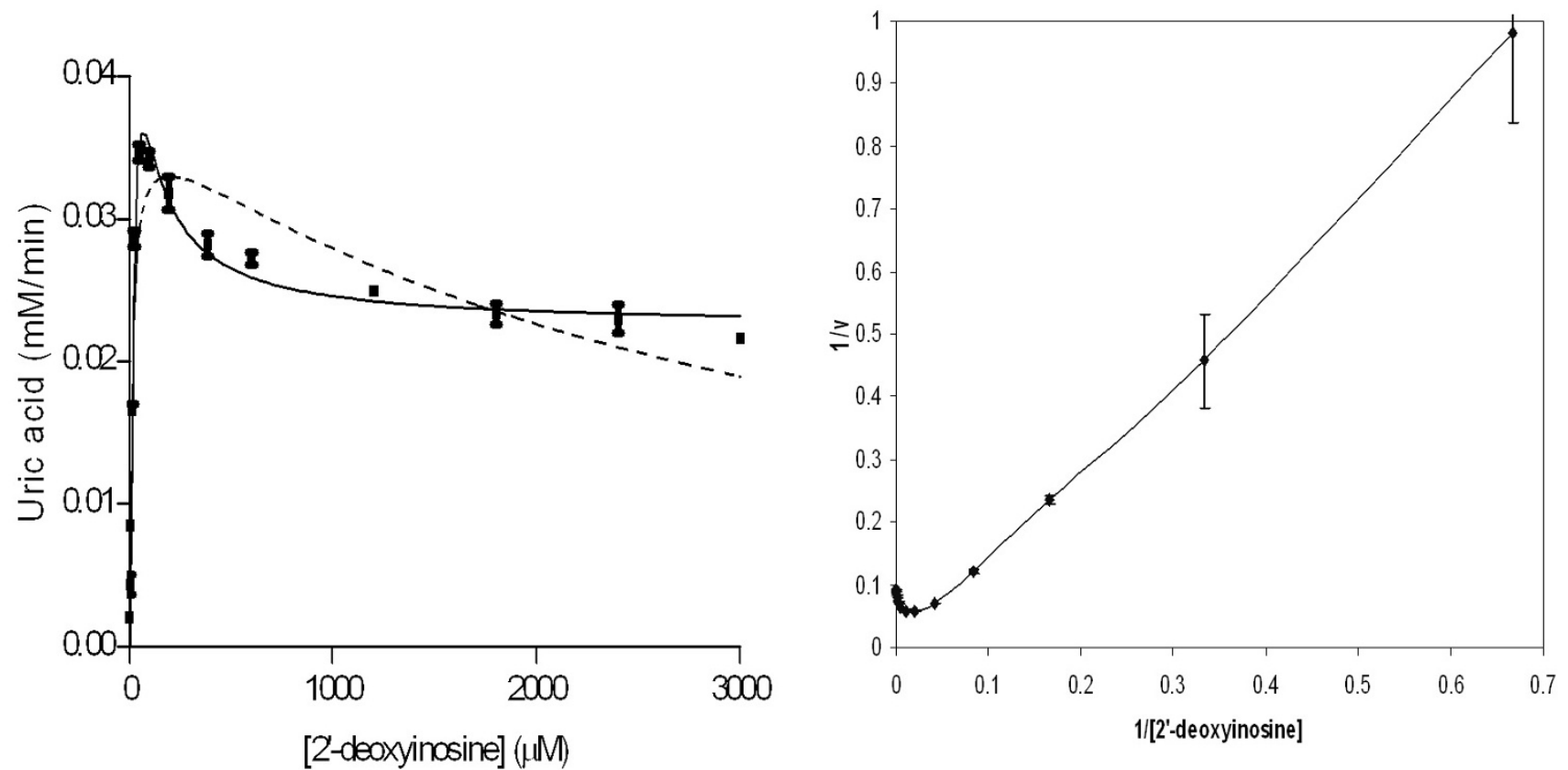

Figure 8

Fitting of initial velocities to different kinetic treatment methods for catalysis of 2'-deoxyinosine by PvPNP. The left $(A)$ shows that application of the substrate activation equation (equation 2 in the text), $v=\left(\left(V_{\max } \cdot \cdot[S]\right)+\left(V_{\max 2} \cdot[S]^{2} / K_{M 2}\right)\right) /$ $\left(K_{M 1}+[S]+\left([S]^{2} / K_{M 2}\right)\right)$, (-) matches the experimental data better than does the theoretical curve from the substrate inhibition equation (---). The reciprocal plot, I/v versus I/[S], with ordinates divided by a factor of 500 (right, B) illustrate the upward curvature at high substrate concentration range, which is similar to an example of a substrate activation case given by [30].

\section{Conclusion}

As expected from sequence homology comparisons, the overall structure and active site of $P v \mathrm{PNP}$ is very similar to those previously described for PfPNP. However, these structures both differ from the crystal structure of PkPNP in which, despite overall conservation of most active site amino acids, a considerably different active site arrangement is observed. This appears to arise from the extended 159-170 loop in PkPNP which protrudes at the dimer interface, changing the subunit association in the dimer and hexamer and, in turn, the binding pocket. It is difficult to conceive, however, that the site could accommodate substrates in this form which may represent an inactive form of the enzyme. The comparability of the $P f P N P$ and $P v$ PNP structures provides confirmation that these are likely to reflect the archetypal Plasmodium forms of the enzyme. The close structural coincidence of their active sites, and their similar overall kinetic profiles, suggest that inhibitors targeting one form of this enzyme are very likely to also prove effective against the other. The key features of these sites are summarised in the proposed generic Plasmodium PNP mechanism shown in Figure 6 and in the schematic in Figure 7.
Although correlation of the active site details of the PfPNP-HRA complex with the expected mechanism is not straightforward, several characteristics suggest the complex is more indicative of a post-transition intermediate conformation along the reaction coordinate rather than a true product complex. In particular, the ribose $\mathrm{C} 1$ ' is located close to equidistant between the base N9 and arsenate $\mathrm{O} 1$, unlike in previous complexes of PfPNP. In this respect the PfPNP-HRA complex is likely to form a good representation of the point of atomic symmetry along the reaction coordinate, and as such may provide a valuable addition to structure-based design efforts.

Despite the remarkably close similarity between PfPNP and $P v P N P$, the response of each enzyme to the 2 -deoxyinosine substrate is differentiated by the observation of an unusual form of substrate activation in PvPNP. Although the mechanism by which this arises is currently unclear, this observation demonstrates that even virtually indistinguishable active sites can respond differently to some substrates - possibly because of electrostatic effects from peripheral regions. Inhibitors that resemble 2'-deoxyinosine might therefore prove less effective against PvPNP. 
Subtle differences such as this may need to be considered during enzyme inhibitor development.

\section{Methods \\ Preparation of recombinant Plasmodium PNPs - cloning, expression, purification}

The gene for PfPNP was exponentially amplified by PCR from genomic DNA and inserted into the pET28a expression vector (Novagen) using procedures essentially as previously described by [9]. Amplification of the PvPNP gene was performed using the same method with the specific primers: - sense: 5'-TCATCCATGGAAGGCGAAATGCAGAGGC-3', and antisense: 5'-CACACTCGAGGTACTTCTTCGCCAATCGGGC-3'. The resulting gene fragment was inserted into the same expression plasmid using the $\mathrm{NcoI}$ and $X h o I$ restriction sites. Both plasmids were transformed into E. coli strain BL21 (DE3) cells (Novagen) for expression. Over-expressed proteins were isolated by nickel affinity chromatography followed by gel filtration using Superdex $^{\mathrm{TM}} 75$ in $150 \mathrm{mM} \mathrm{NaCl}, 50 \mathrm{mM}$ HEPES, pH 7.5. Protein fractions ( $>95 \%$ purity determined by SDS-PAGE analysis) were concentrated to $10 \mathrm{mg} / \mathrm{ml}$ using Vivaspin concentrators (Vivascience) in $100 \mathrm{mM} \mathrm{NaCl}, 100 \mathrm{mM}$ HEPES, pH 7.5.

\section{Crystallisation and structure determination of PvPNP and the PfPNP-complex}

Crystallisation of both PNP enzymes was achieved by vapour diffusion, with conditions established by sparse matrix crystallisation screens, Crystal Screen $1^{\mathrm{Tm}}$ and Crystal Screen2 ${ }^{\text {тм }}$ (Hampton Research). Viable crystals of $P v$ PNP could only be obtained in the absence of nucleosides. The optimised conditions for crystal growth were 5 $\mathrm{mg} / \mathrm{ml}(0.18 \mathrm{mM})$ PvPNP, $18 \%$ PEG $4 \mathrm{~K}, 0.2 \mathrm{M} \mathrm{LiSO}_{4}$ and $0.1 \mathrm{M}$ Tris-HCl, $\mathrm{pH}$ 8.5. Crystals of the PfPNP-hypoxanthine-ribose-arsenate (PfPNP-HRA) complex were obtained by pre-incubating $10 \mathrm{mg} / \mathrm{ml}(0.37 \mathrm{mM})$ PfPNP, $5 \mathrm{mM}$ inosine, and $0.1 \mathrm{M}$ arsenic acid, followed by crystallisation using 4.0 M sodium formate (adjusted to neutral $\mathrm{pH})$ as precipitant.

Diffraction data were collected at the Daresbury SRS synchrotron, station MAD10.1, using monochromatic radiation at wavelengths $1.0745 \AA$ and $1.196 \AA$ respectively for $P v$ PNP and the PfPNP complex. Data were processed using the HKL2000 suite [37] and are summarized in Table 1. Both structures were solved by molecular replacement using the Phaser program [38] in the CCP4 suite [39]. Search models were (1) a monomer of the PkPNP crystal structure (PDB: 2B94) and (2) a monomer (chain A) of the PfPNP-ImmH complex crystal structure (PDB: $\underline{1 N W 4},[6])$, in each case with ligands and solvent molecules removed. The resulting structure solutions were refined using REFMAC5 [39] with manual rebuilding in COOT [40]. For the PfPNP complex, TLS refinement was introduced at the very last refinement step using a tls tensor file calculated from the program TLSMD [41]. Completed structures were verified for geometric correctness with MolProbity [42] and SFCHECK [43]. Refinement statistics are also summarized in Table 1.

Coordinates and structure factors have been deposited in the Protein Data Bank (accession codes: $P v \mathrm{PNP}_{-} \mathrm{SO}_{4}$ : [PDB:3EMV]; PfPNP-HRA complex: [PDB:3ENZ]).

\section{Enzymatic properties of PvPNP compared with PfPNP}

Kinetic assays of the Plasmodium PNPs with various substrates were based on the forward reaction, in which phosphorolysis of the substrates was catalysed by the enzyme in the presence of phosphate, using a coupled reaction with xanthine oxidase and conditions as described previously [9]. The concentration of desalted xanthine oxidase (Sigma) for the coupled reaction in the inosine and 2'deoxyinosine assays was 90 mill-units $/ \mathrm{ml}$, and substrates were included in the following range of concentrations: inosine (Calbiochem) $0.75-3000 \mu \mathrm{M}, 2^{\prime}$-deoxyinosine (Biochemika) $0.75-3000 \mu \mathrm{M}$, guanosine (Sigma) 0.39$150 \mu \mathrm{M}, 2$ '-deoxyguanosine (Sigma) $1.71-200 \mu \mathrm{M}$ and 2amino-6-mercapto-7-methylpurine riboside (MESG, a component of EnzCheck Phophate Assay Kit, Molecular Probes, Invitrogen) $0.5-500 \mu \mathrm{M}$. Measured initial rates were fitted to the classic Michaelis-Menten equation and used for calculation of $K_{\mathrm{M}^{\prime}}, k_{c a t}$ and $k_{\text {cat }} / K_{\mathrm{M}^{\prime}}$ with appropriate corrections made for background rates, using the nonlinear regression facility in the GraphPad Prism software (GraphPad Software, Inc., San Diego, USA). For analysis of the substrate activated form of PvPNP with 2'-deoxyinosine (see discussion), the binding sequence could be described as:

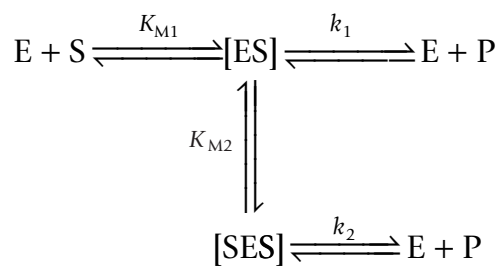

where $\mathrm{E}=$ enzyme, $\mathrm{S}=$ substrate and the rate of the reaction is:

$$
\frac{v}{\mathrm{E}_{0}}=\frac{k_{1} \cdot[\mathrm{ES}]+k_{2} \cdot[\mathrm{SES}]}{[\mathrm{E}]+[\mathrm{ES}]+[\mathrm{SES}]}
$$

and the Michaelis constants, $K_{\mathrm{M}^{\prime}}$ can be approximated as:

$$
K_{\mathrm{M} 1} \approx \frac{[\mathrm{E}] \cdot[\mathrm{S}]}{[\mathrm{ES}]} \text { and } K_{\mathrm{M} 2} \approx \frac{[\mathrm{ES}] \cdot[\mathrm{S}]}{[\mathrm{SES}]}
$$

To simplify the equation for the analysis software, equation 1 was divided by [E]/[E]: 


$$
\frac{v}{\mathrm{E}_{0}}=\frac{k_{1} \cdot \frac{[\mathrm{ES}]}{[\mathrm{E}]}+k_{2} \cdot \frac{[\mathrm{SES}]}{[\mathrm{E}]}}{1+\frac{[\mathrm{ES}]}{[\mathrm{E}]}+\frac{[\mathrm{SES}]}{[\mathrm{E}]}}
$$

And substituting the expressions for the Michaelis constants gives:

$$
\frac{v}{\mathrm{E}_{0}}=\frac{k_{1} \cdot \frac{[\mathrm{S}]}{K_{\mathrm{M} 1}}+k_{2} \cdot \frac{[\mathrm{S}]^{2}}{K_{\mathrm{M} 1} \cdot K_{\mathrm{M} 2}}}{1+\frac{[\mathrm{S}]}{K_{\mathrm{M} 1}}+\frac{[\mathrm{S}]^{2}}{K_{\mathrm{M} 1} \cdot K_{\mathrm{M} 2}}}
$$

This was then multiplied by $K_{\mathrm{M} 1} / K_{\mathrm{M} 1}$ and by $\mathrm{E}_{0}$ :

$$
v=\frac{\mathrm{E}_{0} \cdot k_{1} \cdot[\mathrm{S}]+\mathrm{E}_{0} \cdot k_{2} \cdot \frac{[\mathrm{S}]^{2}}{K_{\mathrm{M} 2}}}{K_{\mathrm{M} 1}+[\mathrm{S}]+\frac{[\mathrm{S}]^{2}}{K_{\mathrm{M} 2}}}
$$

as $V_{\max }=k \cdot \mathrm{E}_{0}$, equation 2 can be derived and to which the data were fitted:

$$
v=\frac{V_{\max 1} \cdot[\mathrm{S}]+V_{\max 2} \cdot \frac{[\mathrm{S}]^{2}}{K_{\mathrm{M} 2}}}{K_{\mathrm{M} 1}+[\mathrm{S}]+\frac{[\mathrm{S}]^{2}}{K_{\mathrm{M} 2}}}
$$

\section{Authors' contributions}

AC performed all of the experimental studies and helped to draft the manuscript. RLB participated in the design of the study, the structural interpretations and comparisons, and prepared the manuscript. Both authors read and approved the final manuscript.

\section{Additional material}

\section{Additional file 1}

Steady-state kinetic data for five purine nucleoside substrates for recombinant PNP enzymes. Table lists enzymatic constants derived in the current study, together with previously published data. Values for $\mathbf{k}_{\text {cat }}$ assume one catalytic site per subunit; values for $K_{\mathrm{M} 1}, K_{\mathrm{M} 2}, k_{\text {cat1 } 1}$ and $k_{\text {cat } 2}$ for 2'-Deoxyinosine refer to Equation 2 in Methods.; Other values from ${ }^{\mathrm{a}}$ [8], b [21], c [22], d [23], e [24], and [25].

Click here for file

[http://www.biomedcentral.com/content/supplementary/14726807-9-42-S1.doc]

\section{Acknowledgements}

We thank Dr. Sittiporn Pattaradilokrat and Associate Prof. Dr. Pongchai Harnyuttanakorn (Chulalongkorn University, Thailand) for kindly providing genomic DNA of Plasmodium vivax, Prof. Anthony Clark (University of Bristol, UK) for help and advice with the kinetic studies, and the staff at the Daresbury SRS and Diamond Light Source for access to data collection facilities. These studies were funded by the award of a DPST scholarship to AC from the Thai government.

\section{References}

I. Gardner MJ, Hall N, Fung E, White O, Berriman M, Hyman RW, Carlton JM, Pain A, Nelson KE, Bowman S, et al:: Genome sequence of the human malaria parasite Plasmodium falciparum. Nature 2002, 4I 9(6906):498-5II.

2. Ting LM, Shi W, Lewandowicz A, Singh V, Mwakingwe A, Birck MR, Ringia EA, Bench G, Madrid DC, Tyler PC, et al.: Targeting a novel Plasmodium falciparum purine recycling pathway with specific immucillins. J Biol Chem 2005, 280( I0):9547-9554.

3. Lewandowicz A, Schramm VL: Transition state analysis for human and Plasmodium falciparum purine nucleoside phosphorylases. Biochemistry 2004, 43(6): 1458-1468.

4. Kline PC, Schramm VL: Pre-Steady-State Transition-State Analysis of the Hydrolytic Reaction Catalyzed by Purine Nucleoside Phosphorylase. Biochemistry 1995, 34(4): I I53-I I 62.

5. Fedorov A, Shi W, Kicska G, Fedorov E, Tyler PC, Furneaux RH, Hanson JC, Gainsford GJ, Larese JZ, Schramm VL, et al.: Transition state structure of purine nucleoside phosphorylase and principles of atomic motion in enzymatic catalysis. Biochemistry 200I, 40(4):853-860.

6. Shi W, Ting LM, Kicska GA, Lewandowicz A, Tyler PC, Evans GB, Furneaux RH, Kim K, Almo SC, Schramm VL: Plasmodium falciparum purine nucleoside phosphorylase: crystal structures, immucillin inhibitors, and dual catalytic function. J Biol Chem 2004, 279(18): 18103-18106.

7. Madrid DC, Ting LM, Waller KL, Schramm VL, Kim K: Plasmodium falciparum purine nucleoside phosphorylase is critical for viability of malaria parasites. J Biol Chem 2008, 283(5 I ):35899-35907.

8. Kicska GA, Tyler PC, Evans GB, Furneaux RH, Kim K, Schramm VL: Transition state analogue inhibitors of purine nucleoside phosphorylase from Plasmodium falciparum. J Biol Chem 2002, 277(5):3219-3225.

9. Schnick C, Robien MA, Brzozowski AM, Dodson EJ, Murshudov GN, Anderson L, Luft JR, Mehlin C, Hol WG, Brannigan JA, et al.: Structures of Plasmodium falciparum purine nucleoside phosphorylase complexed with sulfate and its natural substrate inosine. Acta Crystallogr D Biol Crystallogr 2005, 6 I (Pt 9): I 245-I 254.

10. Price RN, Tjitra E, Guerra CA, Yeung S, White NJ, Anstey NM: Vivax malaria: neglected and not benign. Am J Trop Med Hyg 2007, 77(6 Suppl):79-87.

II. Carlton JM, Adams JH, Silva JC, Bidwell SL, Lorenzi H, Caler E, Crabtree J, Angiuoli SV, Merino EF, Amedeo P, et al:: Comparative genomics of the neglected human malaria parasite Plasmodium vivax. Nature 2008, 455(72 I 4):757-763

12. Ealick SE, Rule SA, Carter DC, Greenhough TJ, Babu YS, Cook W], Habash J, Helliwell JR, Stoeckler JD, Parks RE Jr, et al.: Three-dimensional structure of human erythrocytic purine nucleoside phosphorylase at 3.2 A resolution. J Biol Chem 1990, 265(3): $1812-1820$.

13. Ealick SE, Babu YS, Bugg CE, Erion MD, Guida WC, Montgomery JA, Secrist JA 3rd: Application of crystallographic and modeling methods in the design of purine nucleoside phosphorylase inhibitors. Proc Natl Acad Sci USA I 99 I, 88(24): I I540-I I 544.

14. Mao C, Cook WJ, Zhou M, Koszalka GW, Krenitsky TA, Ealick SE: The crystal structure of Escherichia coli purine nucleoside phosphorylase: a comparison with the human enzyme reveals a conserved topology. Structure 1997, 5(10): I373-1383.

15. Tahirov TH, Inagaki E, Ohshima N, Kitao T, Kuroishi C, Ukita Y, Takio K, Kobayashi M, Kuramitsu S, Yokoyama S, et al.: Crystal structure of purine nucleoside phosphorylase from Thermus thermophilus. Journal of Molecular Biology 2004, 337(5): I 149-1 I60.

16. Koellner G, Bzowska A, Wielgus-Kutrowska B, Luic M, Steiner T, Saenger W, Stepinski J: Open and closed conformation of the E. coli purine nucleoside phosphorylase active center and 
implications for the catalytic mechanism. Journal of Molecular Biology 2002, 3I5(3):35I-37I.

17. Kline PC, Schramm VL: Purine nucleoside phosphorylase. Catalytic mechanism and transition-state analysis of the arsenolysis reaction. Biochemistry 1993, 32(48): 132 12-13219.

18. Grubmeyer C, Cross RL, Penefsky HS: Mechanism of ATP hydrolysis by beef heart mitochondrial ATPase. Rate constants for elementary steps in catalysis at a single site. J Biol Chem 1982, 257(20): I 2092-12100.

19. Bennett EM, Li C, Allan PW, Parker WB, Ealick SE: Structural Basis for Substrate Specificity of Escherichia coli Purine Nucleoside Phosphorylase. J Biol Chem 2003, 278(47):47| I 0-47 I I8.

20. Erion MD, Stoeckler JD, Guida WC, Walter RL, Ealick SE: Purine nucleoside phosphorylase. 2. Catalytic mechanism. Biochemistry 1997, 36(39): I I735-I I 748.

21. Stoeckler JD, Cambor C, Parks RE Jr: Human erythrocytic purine nucleoside phosphorylase: reaction with sugar-modified nucleoside substrates. Biochemistry 1980, I (1): I02-107.

22. Daddona PE, Wiesmann WP, Milhouse W, Chern JW, Townsend LB, Hershfield MS, Webster HK: Expression of human malaria parasite purine nucleoside phosphorylase in host enzyme-deficient erythrocyte culture. Enzyme characterization and identification of novel inhibitors. I Biol Chem 1986, 26 I(25): I I667-II673.

23. Silva RG, Pereira JH, Canduri F, de Azevedo WF Jr, Basso LA, Santos DS: Kinetics and crystal structure of human purine nucleoside phosphorylase in complex with 7-methyl-6-thio-guanosine. Arch Biochem Biophys 2005, 442(I):49-58.

24. Chaudhary K, Ting LM, Kim K, Roos DS: Toxoplasma gondii purine nucleoside phosphorylase biochemical characterization, inhibitor profiles, and comparison with the Plasmodium falciparum ortholog. I Biol Chem 2006, 28 I (35):25652-25658.

25. Taylor EA, Rinaldo-Matthis A, Li L, Ghanem M, Hazleton KZ, Cassera MB, Almo SC, Schramm VL: Anopheles gambiae purine nucleoside phosphorylase: catalysis, structure, and inhibition. Biochemistry 2007, 46(43): I2405-124I5.

26. Shi W, Basso LA, Santos DS, Tyler PC, Furneaux RH, Blanchard JS, Almo SC, Schramm VL: Structures of purine nucleoside phosphorylase from Mycobacterium tuberculosis in complexes with immucillin-H and its pieces. Biochemistry 200I, 40(28):8204-82I5.

27. Schramm VL, Shi W: Atomic motion in enzymatic reaction coordinates. Current Opinion in Structural Biology 200I, I I(6):657-665.

28. Mao C, Cook WJ, Zhou M, Federov AA, Almo SC, Ealick SE: Calf spleen purine nucleoside phosphorylase complexed with substrates and substrate analogues. Biochemistry 1998, 37(20):7|35-7|46.

29. Jordan F, Wu A: Stereoelectronic factors in the binding of substrate analogues and inhibitors to purine nucleoside phosphorylase isolated from human erythrocytes. J Med Chem | 978, 2 | (9):877-882.

30. Frieden C: Treatment of Enzyme Kinetic Data. I. the Effect of Modifiers on the Kinetic Parameters of Single Substrate Enzymes. J Biol Chem 1964, 239:3522-353I.

31. Agarwal RP, Parks RE Jr: Purine nucleoside phosphorylase from human erythrocytes. IV. Crystallization and some properties. J Biol Chem 1969, 244(4):644-647.

32. Agarwal KC, Agarwal RP, Stoeckler JD, Parks RE Jr: Purine nucleoside phosphorylase. Microheterogeneity and comparison of kinetic behavior of the enzyme from several tissues and species. Biochemistry 1975, I4(1):79-84.

33. Whiteley HR, Tahara M: Threonine deaminase of Clostridium tetanomorphum. I. Purification and properties. J Biol Chem I966, 24I(2I):488|-4889.

34. Li L, Luo M, Ghanem M, Taylor EA, Schramm VL: Second-sphere amino acids contribute to transition-state structure in bovine purine nucleoside phosphorylase. Biochemistry 2008 , 47(8):2577-2583.

35. Luo M, Li L, Schramm VL: Remote mutations alter transitionstate structure of human purine nucleoside phosphorylase. Biochemistry 2008, 47(8):2565-2576.

36. Read JA, Winter VJ, Eszes CM, Sessions RB, Brady RL: Structural basis for altered activity of $M$ - and $H$-isozyme forms of human lactate dehydrogenase. Proteins 200I, 43(2): I75-I85.
37. Otwinowski Z, Minor W: Processing of X-ray diffraction data collected in oscillation mode. Meth Enzym 1997, 276:307-326.

38. McCoy AJ: Solving structures of protein complexes by molecular replacement with Phaser. Acta Crystallogr D Biol Crystallogr 2007, 63(Pt I):32-4I.

39. CCP4: Collaborative Computing Project No. 4: The CCP4 suite: programs for protein crystallography. Acta Cryst Section D 1994, 50:760-763.

40. Emsley P, Cowtan K: Coot: Model-Building Tools for Molecular Graphics. Acta Crystallogr D Biol Crystallogr 2004, 60((Pt I2 Pt I)):2126-2I32.

4I. Painter J, Merritt EA: Optimal description of a protein structure in terms of multiple groups undergoing TLS motion. Acta Crystallogr D Biol Crystallogr 2006, 62(Pt 4):439-450.

42. Davis IW, Leaver-Fay A. Chen VB, Block JN, Kapral GJ, Wang X, Murray LW, Arendall WB 3rd, Snoeyink J, Richardson JS, et al.: MolProbity: all-atom contacts and structure validation for proteins and nucleic acids. Nucleic Acids Res 2007:W375-383.

43. Vaguine AA, Richelle J, Wodak SJ: SFCHECK: a unified set of procedures for evaluating the quality of macromolecular structure-factor data and their agreement with the atomic model. Acta Crystallogr D Biol Crystallogr 1999, 55(Pt I): 19|-205.

Publish with Bio Med Central and every scientist can read your work free of charge

"BioMed Central will be the most significant development for disseminating the results of biomedical research in our lifetime. "

Sir Paul Nurse, Cancer Research UK

Your research papers will be:

- available free of charge to the entire biomedical community

- peer reviewed and published immediately upon acceptance

- cited in PubMed and archived on PubMed Central

- yours - you keep the copyright
BioMedcentral 